\title{
Heterozygous Disruption of the DNA Topoisomerase I Gene Confers Cellular Resistance to Camptothecin in Human Cells
}

\author{
Eriko Toyoda, ${ }^{a, b}$ Aya Kurosawa, ${ }^{a}$ Michihiko FujI, ${ }^{a}$ and Noritaka AdACHI*,a \\ ${ }^{a}$ International Graduate School of Arts and Sciences, Yokohama City University; 22-2 Seto, Kanazawa-ku, Yokohama \\ 236-0027, Japan: and ${ }^{b}$ Pharmaceutical Research Laboratories, Nippon Kayaku Co., Ltd.; 3-31-12 Shimo, Kita-ku, Tokyo \\ 115-8588, Japan. Received November 26, 2008; accepted December 19, 2008
}

DNA topoisomerase I (Top1) is a ubiquitous nuclear enzyme that plays essential roles in various cellular processes, such as transcription or replication. Agents that target Top1, involving camptothecin and its derivatives, are among the most effective anticancer drugs used in the clinic. Previous work has suggested that the level of Top1 expression correlates with the cytotoxicity of camptothecin, but no direct evidence has been provided thus far in the context of human cells with a strictly isogenic genetic background. In this study, we perform heterozygous disruption of the Top1 gene (TOP1) by gene targeting in a human pre-B cell line, Nalm-6, which is karyotypically stable and normal for p53 status. We show that the heterozygous loss of the TOP1 gene does confer cellular resistance to camptothecin, to an extent comparable to that observed in the absence of functional p53 protein. Such a tolerance was not observed with other agents that target DNA topoisomerase II. Our results provide direct evidence that human cells with decreased Top1 levels are significantly more resistant to killing by camptothecin than are otherwise isogenic cells.

Key words DNA topoisomerase I; camptothecin; p53; gene targeting; Nalm-6

DNA topoisomerase I (Top1) is a ubiquitous and abundant nuclear enzyme that alters the topological structure of DNA and chromosomes through a transient DNA single-strand break (nick) and subsequent religation of the nick. ${ }^{1-4)}$ The enzyme has been implicated in many aspects of DNA metabolism, including DNA replication and transcription. ${ }^{3,4)}$ Top1 has been of considerable interest to human medicine, because it is an important target for cancer chemotherapy. Top1-targeting agents, as exemplified by camptothecin and its derivatives, are widely used as anticancer drugs in the clinic. ${ }^{6-8)}$ These agents are referred to as "Top1 poisons," since they convert the essential enzyme into a highly cytotoxic DNA-damaging agent through the formation of "cleavage complex" (also called "cleavable complex"), in which Top 1 protein is covalently linked to the $3^{\prime}$-end at the nick. ${ }^{1)}$ Increasing evidence suggests that Top1 cleavage complexes can be converted into irreversible DNA lesions in a replication-dependent or -independent fashion. ${ }^{8-13)}$ The replicationdependent cytotoxic DNA lesions, which arise from collisions between replication forks and Top1 cleavage complexes, involve DNA double-strand breaks and Top1 covalent complexes. ${ }^{9-11,13,14)}$

In addition to Top1, cells express DNA topoisomerase II (Top2), which is also a nuclear enzyme involved in many aspects of DNA metabolism and is an important target for cancer chemotherapy. ${ }^{5)}$ Indeed, Top2-targeting agents, involving etoposide, doxorubicin and mitoxantrone, are among the most effective and widely used anticancer drugs in cancer chemotherapy. ${ }^{15,16)}$ These agents are referred to as "Top2 poisons," as they induce chromosomal double-strand breaks by trapping Top2 cleavage complexes. ${ }^{17,18)}$ Mammalian cells possess two genetically distinct Top 2 isoforms, Top $2 \alpha$ and Top $2 \beta,{ }^{1,19)}$ which are both the target of these Top2-targeting agents. Very recently, we have characterized a novel synthetic benzo $[c]$ phenanthridine alkaloid, NK314, that exhibits strong antitumor activity and found that this agent acts as an $\alpha$-isoform-specific Top2 poison in living mammalian cells. ${ }^{20)}$

Previous work has suggested that the level of Top1 expres- sion correlates with the cytotoxicity of camptothecin. ${ }^{6,21,22)}$ In fact, in mouse embryonic stem cells, increased camptothecin resistance was successfully employed to select for correct gene disruption at the Top1 locus. ${ }^{21)}$ In the context of human somatic cell lines, however, there has been no such evidence because of the lack of a series of mutant cell lines in a strictly isogenic genetic background. In this study, we, for the first time, generate human cells with a genetically engineered TOP1 allele. Specifically, we generate human TOP1 +/cells by gene targeting to perform phenotypic analysis of the mutant. Our results have significant implications for our understanding of camptothecin tolerance as well as for the future cancer treatment using Top1-targeting drugs.

\section{MATERIALS AND METHODS}

Cell Culture The human pre-B cell line Nalm-6 and its derivatives were cultured in ES medium (Nissui Seiyaku Co., Tokyo, Japan) supplemented with 10\% calf serum (Hyclone, Logan, UT, U.S.A.) and $50 \mu \mathrm{m} 2$-mercaptoethanol at $37^{\circ} \mathrm{C}$ in a humidified atmosphere containing 5\% $\mathrm{CO}_{2}$. TP53 -/Nalm-6 cells have previously been described. ${ }^{23}{ }^{2}$

Topoisomerase Inhibitors Camptothecin was purchased from Sigma-Aldrich (St. Louis, MO, U.S.A.). Etoposide and NK314 were synthesized at Nippon Kayaku (Tokyo, Japan). Camptothecin and etoposide were dissolved in dimethyl sulfoxide and NK314 in distilled water. All the drugs were stored frozen in aliquots at $-20^{\circ} \mathrm{C}$.

Southern and Western Blot Analysis Southern blot analysis was performed as described previously. ${ }^{24)}$ Briefly, $10 \mu \mathrm{g}$ of genomic DNA were digested with $B g l \mathrm{II}$, electrophoresed in a $0.8 \%$ agarose gel, and transferred to a Hybond- $\mathrm{N}^{+}$membrane (GE Healthcare Bio-Sciences, Piscataway, NJ, U.S.A.), followed by Southern hybridization with a ${ }^{32} \mathrm{P}$-labeled probe. Western blot analysis was carried out as described previously. ${ }^{25)}$ Anti-Top $2 \alpha$, -Top $2 \beta$, and -Ku70 antibodies were purchased from BD Transduction Laboratories (Bedford, MA, U.S.A.). Anti-Top1 antibody was purchased 
from Santa Cruz Biotechnology (Santa Cruz, CA, U.S.A.) and anti-actin antibody from Sigma-Aldrich. Levels of expression were quantified using a Fuji image analyzer LAS1000UVmini and a MultiGauge software (Fuji Film Co., Tokyo, Japan).

Gene Targeting in Human Nalm-6 Cells The targeting vector pTOP1-Hyg was constructed by using a simplified construction method based on the MultiSite Gateway ${ }^{\circledR}$ Technology (Invitrogen, Carlsbad, CA, U.S.A.) as described. ${ }^{26,27)}$ For this purpose, human TOP1 genomic fragments were obtained by polymerase chain reaction (PCR) amplification with ExTaq DNA polymerase (Takara Bio Inc., Otsu, Japan) from Nalm-6 genomic DNA using primers Top1-5armF (5' GCCAAAGGGAAGCCATTTCGATG-3') and Top1-5armR (5'-GCAGCTGGGCAGAGTACCTCACAC-3') for the 5'arm, and Top 1-3armF (5'-CCACCATATGAGCCTCTTCCAGAG-3') and Top1-3armR (5'-CTTCCTCTGCTTTGGGGCTCAGC- $3^{\prime}$ ) for the $3^{\prime}$-arm (note that all the primers contain an $a t t \mathrm{~B}$ sequence at their $5^{\prime}$-end, though not indicated). The targeting vector was linearized with the I-SceI restriction enzyme, and transfected into wild-type Nalm- 6 cells, as previously described. ${ }^{23,24,26-28)}$ Briefly, $4 \times 10^{6}$ cells were electroporated with $4 \mu \mathrm{g}$ of the linearized vector, cultured for $22-24 \mathrm{~h}$, and replated at a density of $0.5-1 \times 10^{6}$ per 90 $\mathrm{mm}$ dish into agarose medium containing $0.4 \mathrm{mg} / \mathrm{ml}$ hygromycin B (Wako Pure Chemical, Osaka, Japan). After a 2-3 week incubation at $37^{\circ} \mathrm{C}$, hygromycin-resistant colonies were isolated, and genomic DNA was prepared from each clone and subjected to Southern blot analysis.

Sensitivity Assays Clonogenic survival assays using Nalm-6 and its derivative cell lines were performed as described previously. ${ }^{24)}$ Briefly, exponentially growing cells were plated at $10^{2}-10^{5}$ cells/dish into $60-\mathrm{mm}$ dishes containing $5 \mathrm{ml}$ of agarose medium with various concentrations of topoisomerase inhibitors. After a 2-3 week incubation at $37^{\circ} \mathrm{C}$, visible colonies were counted, and the percent survival was determined by comparing the number of surviving colonies to untreated controls. Statistical significance was determined using the paired Student's $t$-test.

\section{RESULTS AND DISCUSSION}

Generation of TOP1 +/- Human Cells We have recently shown that the human pre-B cell line Nalm-6 is highly proficient for gene targeting by homologous recombination, which, in general, barely occurs in cultured mammalian cells. $^{23)}$ To generate TOP1 $+/-$ Nalm-6 cells, we transfected linearized targeting vector pTOP1-Hyg (Fig. 1A) into wildtype cells. Targeted disruption of one allele of the TOP1 gene (i.e., heterozygous gene disruption) was confirmed by Southern and Western blot analysis (Figs. 1B, C). Of note, Top1 levels in the TOP $1+/$ - cells were decreased to $c a .70 \%$ that of wild-type cells. The heterozygous gene disruption did not affect expression levels of Top 2 proteins (Top $2 \alpha$ and Top $2 \beta$ ).

Growth Properties of TOP1 +/- Cells We compared the growth rate of TOP1 $+/-$ cells with that of wild-type cells and found no significant difference in growth rate between the two cell lines (Fig. 2A; the doubling-times of wildtype and TOP $1+/-$ cells were both $c a .20 \mathrm{~h}$ ). Additionally, there was little or no difference in plating efficiency between wild-type and TOP1 +/- cells (Fig. 2B). These results show
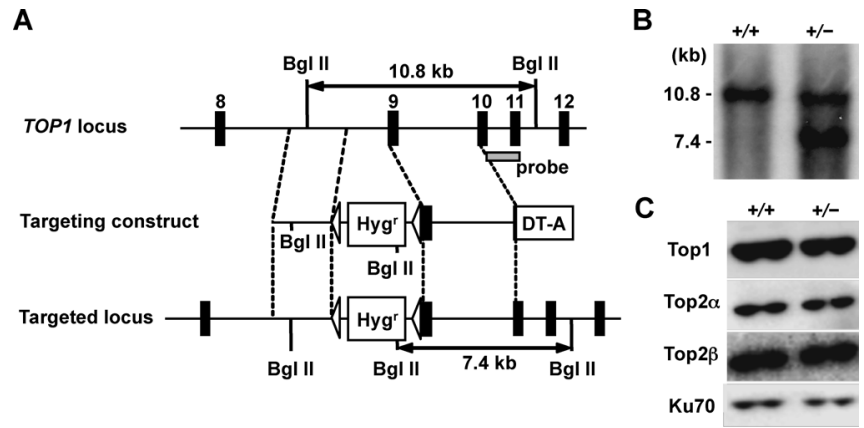

Fig. 1. Targeted Disruption of the Human TOP1 Gene

(A) Scheme for $T O P 1$ gene targeting. The targeting vector was designed to replace exon 9 with a hygromycin-resistance $\left(\mathrm{Hyg}^{\mathrm{r}}\right)$ gene. The figure is not drawn to scale. Triangles represent lox $\mathrm{P}$ sequences. DT-A, a gene encoding diphtheria toxin A fragment. (B) Southern blot analysis of BglII-digested genomic DNA from wild-type $(+/+)$ and TOP1 heterozygous $(+/-)$ cells using the probe shown in (A). (C) Western blot analysis for Top1, Top $2 \alpha$, Top $2 \beta$ and Ku70 of wild-type $(+/+)$ and TOP1 heterozygous $(+/-)$ cells. Whole cell extract from $1 \times 10^{5}$ cells was loaded on a $7.5 \%$ SDS-polyacrylamide gel. Levels of expression were quantified using an image analyzer. Ku70 served as a loading control.
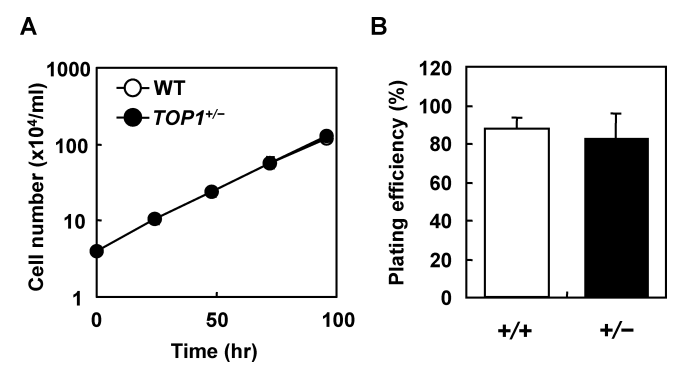

Fig. 2. Growth Properties of TOP1 +/- Nalm-6 Cells

(A) Growth curves of wild-type and TOPI heterozygous cells. Data are the mean \pm S.D. of three independent experiments. All error bars fall within the symbols. (B) Plating efficiencies of wild-type $(+/+)$ and TOPI heterozygous $(+/-)$ cells. Data are the mean \pm S.D. of eleven independent experiments.

that heterozygous disruption of TOP1 in Nalm-6 causes little or no growth defects under ordinary culture conditions.

Increased Camptothecin Resistance in TOP1 +/- Cells We next performed clonogenic survival assays in the presence of camptothecin to examine the sensitivity of TOP1 $+/-$ cells to this drug. As shown in Fig. 3A, the mutant cells showed increased resistance to camptothecin relative to wildtype cells, and the differences were statistically significant (e.g., $p<0.005$ at $4 \mathrm{~nm}$ ). Additionally, concentrations that achieve a $90 \%$ inhibition in survival were statistically different $(4.1 \pm 0.2 \mathrm{~nm}$ for wild-type $v s$. $5.2 \pm 0.2 \mathrm{~nm}$ for TOP $1+/-$, $p<0.05)$. By contrast, the mutant cells did not show increased resistance to the Top2 poisons etoposide and NK314 (Figs. 3B, C). These observations clearly show that decreased levels of Top1 expression result in cellular resistance to camptothecin. The data also confirm that NK314 does not target Top1 in living cells. ${ }^{28)}$

Recently, Burgess et al. reported that murine cells with decreased Top1 expression showed increased sensitivity to Top2 poisons. $^{29)}$ In addition, Miao et al. used siRNAs to reduce Top1 expression $c a$. 5-fold in human cell lines, and showed that those Top1 knockdown cells were $c a$. 2-fold more sensitive to Top 2 poisons. ${ }^{30)}$ In the present study, we did not observe an increased sensitivity to etoposide in TOP1 $+/-$ cells. It is possible that since our mutant cell line retained as high as $c a$. 70\% that of wild-type Top1 levels, func- 
A

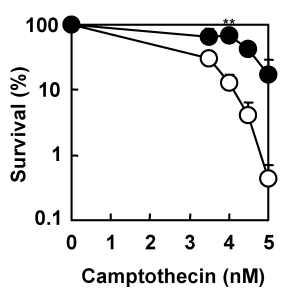

B

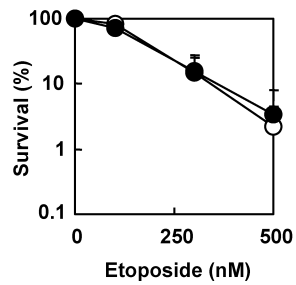

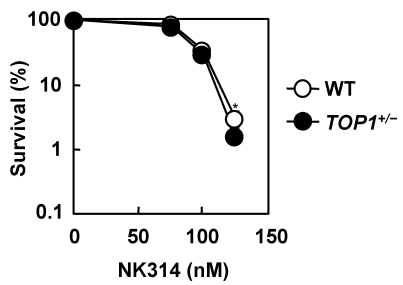

Fig. 3. Sensitivity of Human TOP1 +/- Cells to Camptothecin, Etoposide, and NK314

Wild-type and TOP1 heterozygous cells were treated with the indicated concentrations of camptothecin (A), etoposide (B), and NK314 (C) and allowed for colony formation, as described in Materials and Methods. Data are the mean \pm S.D. of three to four independent experiments $(* p<0.05$, $* * p<0.005)$. Where absent, error bars fall within symbols.

A

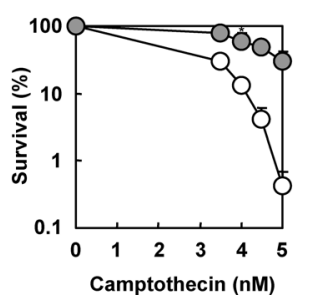

B

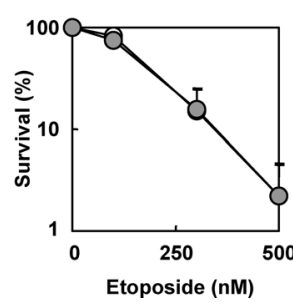

C

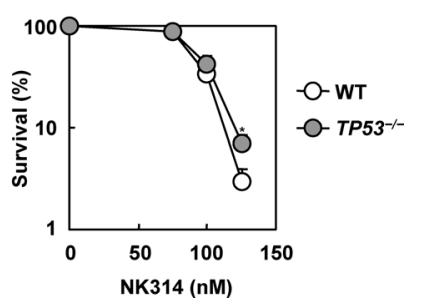

Fig. 4. Sensitivity of Human TP53 -/- Cells to Camptothecin, Etoposide, and NK314

Wild-type and TP53-knockout cells were treated with the indicated concentrations of camptothecin (A), etoposide (B), and NK314 (C) and allowed for colony formation, as described in Materials and Methods. Data are the mean \pm S.D. of three to four independent experiments $(* p<0.05)$. Where absent, error bars fall within symbols.

tional compensation by Top2 was not prominent enough to confer increased sensitivity to etoposide. We note, however, that our TOP $1+/-$ cells were slightly but reproducibly more sensitive to NK314 than wild-type cells (e.g., $p<0.05$ at $125 \mathrm{~nm})$. In this regard, it is intriguing that the study by Miao et al. suggested that Top $2 \alpha$ (and not Top $2 \beta$ ) could compensate for the reduced Top 1 function. ${ }^{30)}$ Thus, reduced Top 1 levels may lead to increased sensitivity to those agents that target Top $2 \alpha$, not Top $2 \beta$.

Increased Camptothecin Resistance in TP53 - /- Cells The increased camptothecin resistance we observed in the TOP $1+/-$ mutant prompted us to employ TP53 knockout human cells to evaluate the significance of this tolerance, as TP53 is the most frequently mutated gene in human cancers and is reported to impact on the cellular sensitivity to various genotoxic anticancer agents. ${ }^{31,32)}$ We previously generated and characterized TP53 - / - Nalm-6 cells, which in fact exhibited increased resistance to ionizing radiation, with a concomitant decrease in the number of dying cells. ${ }^{33)}$ We thus examined the sensitivity of TP53 - / - cells to camptothecin. As shown in Fig. 4A, the mutant cells showed significantly increased resistance to camptothecin relative to wild-type cells $(p<0.05$ at $4 \mathrm{~nm})$. It should be noted that the level of the tolerance was similar or comparable to that observed in the TOP1 +/- mutant (see Fig. 3A). Consistent with our previous report, ${ }^{33)}$ TP53 $-/-$ cells did not show increased resistance to the Top2 poison etoposide, though this seemed not to be the case for NK314 (Figs. 4B, C).

Concluding Remarks and Future Perspectives In the present study, we have conducted genetic analyses using gene-knockout human cell lines to investigate cellular sensitivity to camptothecin. Indeed, this is the first report on the generation of a human mutant cell line with a genetically deleted TOP1 allele. The heterozygous disruption of the human TOP1 gene resulted in a significant decrease in Top1 levels and conferred cellular resistance to the Top1 poison camptothecin. Our results confirm the notion that the level of Top1 expression correlates with camptothecin cytotoxicity, and do provide direct supporting evidence in the context of human somatic cells. Most importantly, the level of camptothecin resistance of TOP $1+/-$ cells was similar or comparable to that observed with a TP53 - / - mutant, implying that the level of Top1 expression is a more critical determinant of cellular sensitivity to Top1 poisons than is the p53 status. Our results also suggest that cells with decreased levels of Top1 expression in a p53-negative background would be even more resistant to camptothecin, though this may await further work with a double-knockout cell line lacking both Top1 and p53.

Intriguingly, we previously reported that loss of non-homologous end-joining (NHEJ), a major repair pathway for repair of DNA double-strand breaks, confers camptothecin resistance in vertebrate cells. ${ }^{34)}$ Additionally, we have recently found that human cells lacking both p53 and DNA ligase IV (a critical NHEJ protein) are more resistant to camptothecin than either single mutant (our unpublished results), thus highlighting the notion that the repair of Top1-mediated DNA damage is an important determinant for the cellular responses to Top1 poisons and for the selectivity of these agents for cancer cells. Collectively, it is conceivable that multiple factors (e.g., Top1, p53, and NHEJ proteins) impact on cellular sensitivity to camptothecin. It will thus be interesting to examine whether one or more of these factors may be compromised in certain cancer cells that have acquired resistance to Top1 poisons. Finally, it should be emphasized that since the human cell mutants described herein have been created by targeted gene disruption, the isogenicity between the cell lines is otherwise completely retained. Hence, these human cell mutants will be valuable for studying the contribution of topoisomerases to the cytotoxicity of potential anticancer agents. 
Acknowledgments This work was supported in part by grants from Yokohama City University (Strategic Research Project, K19009 and K20005), and by a Grant-in-Aid from the Ministry of Education, Culture, Sports, Science and Technology (MEXT) of Japan.

\section{REFERENCES}

1) Champoux J. J., Annu. Rev. Biochem., 70, 369-413 (2001).

2) Pommier Y., Pourquier P., Fan Y., Strumberg D., Biochim. Biophys. Acta, 1400, 83-105 (1998).

3) Wang J. C., Nat. Rev. Mol. Cell Biol., 3, 430-440 (2002).

4) Wang J. C., Annu. Rev. Biochem., 65, 635-692 (1996).

5) Li T. K., Liu L. F., Annu. Rev. Pharmacol. Toxicol., 41, $53-77$ (2001).

6) Pommier Y., Nat. Rev. Cancer, 6, 789-802 (2006).

7) Hsiang Y. H., Hertzberg R., Hecht S., Liu L. F., J. Biol. Chem., 260, 14873-14878 (1985).

8) Pourquier P., Pommier Y., Adv. Cancer Res., 80, 189-216 (2001).

9) Strumberg D., Pilon A. A., Smith M., Hickey R., Malkas L., Pommier Y., Mol. Cell. Biol., 20, 3977-3987 (2000).

10) Barrows L. R., Holden J. A., Anderson M., D’Arpa P., Mutat. Res., 408, 103-110 (1998).

11) Nitiss J. L., Beck W. T., Eur. J. Cancer, 32A, 958-966 (1996).

12) Hsiang Y. H., Lihou M. G., Liu L. F., Cancer Res., 49, 5077-5082 (1989).

13) Desai S. D., Zhang H., Rodriguez-Bauman A., Yang J. M., Wu X., Gounder M. K., Rubin E. H., Liu L. F., Mol. Cell. Biol., 23, 23412350 (2003).

14) Pouliot J. J., Robertson C. A., Nash H. A., Genes Cells, 6, 677-687 (2001).

15) Osheroff N., Biochemistry, 28, 6157-6160 (1989).

16) Cummings J., Smyth J. F., Ann. Oncol., 4, 533-543 (1993).

17) Nitiss J. L., Wang J. C., Mol. Pharmacol., 50, 1095-1102 (1996).

18) Liu L. F., Annu. Rev. Biochem., 58, 351-375 (1989).
19) Austin C. A., Marsh K. L., Bioessays, 20, 215-226 (1998).

20) Onda T., Toyoda E., Miyazaki O., Seno C., Kagaya S., Okamoto K., Nishikawa K., Cancer Lett., 259, 99-110 (2008).

21) Morham S. G., Kluckman K. D., Voulomanos N., Smithies O., Mol. Cell. Biol., 16, 6804-6809 (1996).

22) Kapoor R., Slade D. L., Fujimori A., Pommier Y., Harker W. G., Oncol. Res., 7, 83-95 (1995).

23) Adachi N., So S., Iiizumi S., Nomura Y., Murai K., Yamakawa C., Miyagawa K., Koyama H., DNA Cell Biol., 25, 19-24 (2006).

24) So S., Adachi N., Lieber M. R., Koyama H. J. Biol. Chem., 279, 55433-55442 (2004).

25) Uegaki K., Adachi N., So S., Iiizumi S., Koyama, H., DNA Repair, 5, 303-311 (2006).

26) Iiizumi S., Nomura Y., So S., Uegaki K., Aoki K., Shibahara K., Adachi N., Koyama H., Biotechniques, 41, 311-316 (2006).

27) Adachi N., Kurosawa A., Koyama H., Methods Mol. Biol., 435, 17 29 (2008).

28) Toyoda E., Kagaya S., Cowell I. G., Kurosawa A., Kamoshita K., Nishikawa K., Iiizumi S., Koyama H., Austin C. A., Adachi N., J. Biol. Chem., 283, 23711-23720 (2008).

29) Burgess D. J., Doles J., Zender L., Xue W., Ma B., McCombie W. R., Hannon G. J., Lowe S. W., Hemann M. T., Proc. Natl. Acad. Sci. U.S.A., 105, 9053-9058 (2008).

30) Miao Z. H., Player A., Shankavaram U., Wang Y. H., Zimonjic D. B., Lorenzi P. L., Liao Z. Y., Liu H., Shimura T., Zhang H. L., Meng L. H., Zhang Y. W., Kawasaki E. S., Popescu N. C., Aladjem M. I., Goldstein D. J., Weinstein J. N., Pommier Y., Cancer Res., 67, 8752-8761 (2007).

31) Levine A. J., Momand J., Finlay C. A., Nature (London), 351, 453456 (1991).

32) Hollstein M., Sidransky D., Vogelstein B., Harris C. C., Science, 253, 49-53 (1991).

33) So S., Adachi N., Koyama H., DNA Cell Biol., 26, 517-525 (2007).

34) Adachi N., So S., Koyama H., J. Biol. Chem., 279, 37343-37348 (2004). 